\section{Perinatal circumstances and risk of offspring suicide}

\author{
Birth cohort study \\ DANIEL V. RIORDAN, SIVASUBR AMANIAM SELVARAJ, CAMERON STARK \\ and JULIE S. E. GILBERT
}

\begin{abstract}
Background A higher risk of suicide has been associated with low birth weight in one study, but not yet replicated. Higher birth order has been associated with selfharm, but not with suicide.
\end{abstract}

\begin{abstract}
Aims To examine the relationship between perinatal circumstances and subsequent young adult suicide in Scotland.
\end{abstract}

\section{Method Using linked data from the Scottish Morbidity Record and Scottish death records, a birth cohort of I 061830 people was followed-up for a mean of 25.1 years. Data were analysed using Cox regression.}

\section{Results Higher maternal parity, younger maternal age ( $<25$ years), non- professional parental occupations and low birth weight $(<2500 \mathrm{~g}$ ) were independently associated with higher suicide risk of offspring as young adults. There was no independent association with gestational age.}

\section{Conclusions Our findings provide support for the influence of maternal circumstance and foetal experience on subsequent mental health.}

\section{Declaration of interest None.} Funding by the Scottish Chief Scientist Office.
Perinatal and early childhood factors may exert a lifelong influence on mental health. There is evidence that antenatal maternal stress may adversely influence the psychological development of offspring (Glover \& O'Connor, 2002). Low birth weight (Thompson et al, 2001) and maternal malnutrition in pregnancy (Brown et al, 2000) have been associated with a higher rate of affective disorders in adulthood. Although Li et al (2003) had failed to demonstrate a significant association between birth weight and teenage suicide, a recent Swedish study (Mittendorfer-Rutz et al, 2004) did find low birth weight and younger maternal age to be associated with offspring suicide as young adults. They also found higher maternal parity to be a risk factor for attempted, but not completed, suicide. This study uses population-based Scottish birth and death records to test the hypothesis, in a UK population, that perinatal factors associated with young adult suicide include low birth weight, premature birth, maternal parity and maternal age.

\section{METHOD}

This study used electronic linkage of datasets held by Information Services of the NHS National Services Scotland. The datasets linked contain maternity hospital discharge records, which are part of the Scottish Morbidity Record, and death certificate details from the General Register Office for Scotland.

The study was approved by the Information Services' Privacy and Advisory Committee.

\section{Maternity records}

A birth cohort was identified using the Scottish Morbidity Record's maternity records. These have been kept since 1969, recording details on births in Scottish National Health Service hospitals as well as some home births. In 1969 they covered approximately $64 \%$ of registered births, rising to over $95 \%$ by 1975 . The Scottish Morbidity Record's maternity records include data on birth weight, gestational age at birth, maternal age, maternal parity and paternal occupation or, where paternal details were unavailable, maternal occupation.

The cohort to be studied was defined as all individuals born alive between 1 January 1969 and 31 December 1986, for whom an electronic Scottish Morbidity Record maternity record pertaining to their birth could be identified. Infants who had died before their mother was discharged from hospital were excluded, as were multiple pregnancies, because low-birthweight and preterm infants are likely to be overrepresented in these groups.

\section{Linkage to death records}

Outcome was determined from the existence or otherwise of a General Register Office for Scotland death record. These records include details on the cause of death and exist in a standard electronic format from 1981 to the present.

For the purpose of this study, Scottish Morbidity Record maternity records were linked to General Register Office for Scotland death records. The maternity record holds identifying information of the mother (surname, previous surname and forenames), together with the date of birth and gender of the baby. The death record holds the date of birth and gender of an individual together with identifying information of their mother (surname, previous surname and forenames). The mother's identifying information, together with the date of birth and gender of the offspring, allowed us to link these records together to create a data-set containing both birth and death information.

From the full General Register Office for Scotland death file, records pertaining to individuals born between 1969 and 1986 were extracted and linked to the Scottish Morbidity Record maternity file by probability matching (Kendrick \& Clarke, 1993). This took account of changes in items of person-identifying information by quantifying levels of discrepancy. Algorithms were used to compare individual items on each record, and an accumulative linkage weight was calculated. Clerical checking established a threshold that minimised the occurrence of false links. 
All deaths registered in Scotland between 1 January 1981 and 31 December 2003, occurring in a member of the birth cohort, were identified. As suicide before the age of 12 years is very rare (Hawton \& James, 2005), deaths before 1981, even in the eldest members of the cohort, were unlikely to have been suicides.

Deaths were classified, for the purpose of this study, as resulting from self-inflicted injury (ICD codes E950-959, X60-84, Y87.0), an event of undetermined intent (ICD codes E980-989, Y10-34, Y87.2) or from other causes. ICD-9 (World Health Organization, 1977) coding was used from 1981 to 1999, ICD-10 (World Health Organization, 1992) from 2000 onwards. To allow for any underrecording, as suicide deaths may be misclassified (Kelly \& Bunting, 1998), it is conventional for the General Register Office for Scotland, when calculating figures for suicide, to combine deaths classified as undetermined with those recorded as self-inflicted injury. This convention was adopted by this study in order to increase comparability with previous similar studies (Hall et al, 1998; Mittendorfer-Rutz et al, 2004).

All members of the birth cohort could therefore be classified as cases of suicide (death by self-inflicted injury or undetermined death, registered in Scotland), cases of death from other causes or non-cases.

\section{Statistical analysis}

The following covariates were treated categorically and entered as indicator variables: birth weight $(<2500 \mathrm{~g}, 2500 \mathrm{~g}-$ $3249 \mathrm{~g}, 3250 \mathrm{~g}-3749 \mathrm{~g}, 3750-4499 \mathrm{~g}$ and $>4500 \mathrm{~g})$; mother's age in years (15-19, 20-24, 25-29, 30-34 and 35-50); mother's parity $(0,1,2,3$ or above); and parental occupation (professional, skilled and unskilled). The birth weight categories, the same as those used by Mittendorfer-Rutz et al (2004), were chosen in order to allow comparison with that study. In separate analyses, birth weight was treated both as a continuous variable and as a dichotomous variable, according to whether it was above or below $2500 \mathrm{~g}$. Similarly, although in the main analysis gestational age was treated as a continuous variable, in a separate analysis it was also treated as a dichotomous variable, according to whether the birth was pre- or post-term (37 weeks). Gender was entered as a dichotomous variable.

As the birth cohort was recruited over a long time, during which there may have been significant secular trends in some variables such as parity, it was subdivided into three sub-cohorts, or age bands, according to the period of birth. These periods were 1969-1974, 1975-1980 and 1981-1986. Period of birth was controlled for in the multivariable analysis, by entering it as a categorical variable.

In all, 1120289 cohort members were identified, representing over $86 \%$ of the births registered in Scotland between 1969 and 1986. A number of records were excluded from the analysis because of details that suggested misclassifications. These were gestational age at birth less than 26 weeks or greater than 45 weeks, maternal age at birth less than 15 years or greater than 50 years, and birth weight below $750 \mathrm{~g}$ or above $6000 \mathrm{~g}$. Also excluded were records that were linked to a death (after 1981) before the age of 10 years. This left 1061830 cases for analysis.

Stata version 8.0 for Windows was used for the analyses of the anonymised data. Suicide and death from other causes were entered as outcome variables. Univariable and multivariable hazard ratios were estimated with Cox proportional hazard regression (using age as the time axis) for the periods from birth to whichever came first: suicide or death from another cause. The proportional hazard assumption was adequately tested.

In the univariable analyses we looked at the effect of each independent categorised or dichotomised variable on both the risk of suicide and the risk of death from other causes. Hazard ratios with $95 \%$ confidence intervals (CIs) and $P$-values were derived from the Wald test for the categorised variables in relation to the reference category. Covariates with $P<0.05$ at the univariable analysis were included in the multivariable model development (results shown as overall $P$-values). For each variable studied, a population-attributable fraction for suicide was calculated.

\section{RESULTS}

Of the 1061830 birth cohort records analysed, 10814 were registered as having died in Scotland before 31 December 2003. Self-inflicted injury was recorded as the cause of death in 1053 of cases and undetermined intent in 411 , so that 1464 cases were identified as suicide; 1166 of these were male and 298 were female. Of those who died by suicide aged 16 years or over, 1123 were male and 275 were female. This suggests a young-adult suicide rate in Scotland in the order of 225 per million per year for males and 58 per million per year for females. Univariable and multivariable results pertaining to deaths from suicide are given in Table 1.

\section{Gender}

Males were significantly more likely to have died by suicide, with a hazard ratio (HR) of 3.82 (95\% CI 3.36-4.34).

\section{Maternal parity}

A significant association was found between maternal parity and subsequent offspring suicide. Compared with the reference category of maternal nulliparity (firstborns), individuals born to women who had had one or two previous completed pregnancies (para 1 or 2 ) were more likely to have died by suicide $(\mathrm{HR}=1.6,95 \% \mathrm{CI}$ para 1 1.41-1.84, 95\% CI para 2 1.36-1.93). This risk increased for para 3 or above $(\mathrm{HR}=2.76,95 \%$ CI $2.29-3.31)$. The population-attributable fraction for the effect of maternal parity on suicide was 0.24 .

This association was observed across all three periods of birth, and in both genders. When the relationship between maternal parity and offspring suicide was analysed separately for each category of parental occupation, a similar association was observed in all three occupation categories (professional, skilled and unskilled).

An association was also found between rising maternal parity and increased risk of offspring death from other causes (para 1: $\mathrm{HR}=1.25$, 95\% CI 1.16-1.34; para 3: $\mathrm{HR}=1.82$, 95\% CI 1.64-2.02).

\section{Maternal age}

Individuals born to women 24 years old or younger were at significantly greater risk of subsequent suicide compared with the reference group of 25-29 years. This risk was higher among those born to women aged 19 years or under $(\mathrm{HR}=2.0,95 \% \mathrm{CI}$ 1.66-2.40). In the offspring of mothers aged 20-24 years, although the risk was not as high, it remained significant $(\mathrm{HR}=1.4,95 \%$ CI 1.23-1.61). No effect of maternal age was observed from 25 years upwards. The effect of maternal age was similar across the three periods of birth, and in both genders. The populationattributable fraction for the influence of young maternal age was 0.20 . 
Table I Univariable and multivariable hazard ratios for suicide by perinatal characteristics, with gestational age as a continuous variable and birth weight, maternal age and maternal parity as categorical variables

\begin{tabular}{|c|c|c|c|c|c|}
\hline \multirow[t]{2}{*}{ Perinatal characteristic } & \multicolumn{3}{|c|}{ Univariable $(n=|06| 830)$} & \multicolumn{2}{|c|}{ Multivariable $(n=|06| 830)$} \\
\hline & Suicides/non-cases, $n$ & Hazard ratio $(95 \% \mathrm{Cl})$ & $P$ & Hazard ratio $(95 \% \mathrm{Cl})$ & $P$ \\
\hline \multicolumn{6}{|l|}{ Gender } \\
\hline Female & $298 / 515739$ & I & & 1 & \\
\hline Male & $1166 / 544627$ & 3.71 (3.27-4.22) & 0.001 & $3.82(3.36-4.34)$ & 0.001 \\
\hline \multicolumn{6}{|l|}{ Birthweight (g) } \\
\hline$<2500$ & $90 / 54735$ & $1.22(0.98-I .53)$ & 0.075 & 1.35 (I.05-I.72) & 0.017 \\
\hline $2500-3249$ & $552 / 390855$ & $1.02(0.91-1.15)$ & 0.696 & I.II (0.98-I.25) & 0.099 \\
\hline $3250-3749$ & $548 / 404240$ & I & & I & \\
\hline $3750-4499$ & $261 / 198164$ & $0.99(0.85-I .14)$ & 0.856 & $0.88(0.76-1.03)$ & 0.103 \\
\hline$>4500$ & $13 / 12372$ & $0.79(0.45-\mathrm{I} .37)$ & 0.396 & $0.62(0.36-1.07)$ & 0.088 \\
\hline Gestational age (weeks) & $1464 / 1060366$ & $0.98(0.96-1.0 \mathrm{I})$ & 0.248 & $1.02(0.99-1.05)$ & 0.196 \\
\hline \multicolumn{6}{|l|}{ Maternal age (years) } \\
\hline $15-19$ & $212 / 111698$ & I.64 (I.39-I.94) & 0.001 & $2.00(1.66-2.4 I)$ & 0.001 \\
\hline $20-24$ & $534 / 354264$ & $1.29(1.13-1.47)$ & 0.001 & $\mathrm{I} .40(1.23-1.60)$ & 0.001 \\
\hline $25-29$ & $398 / 357959$ & I & & 1 & \\
\hline $30-34$ & $221 / 169652$ & $\mathrm{I} .20(\mathrm{I} .02-\mathrm{I} .4 \mathrm{I})$ & 0.030 & $1.06(0.89-1.25)$ & 0.524 \\
\hline $35-45$ & $99 / 66793$ & I. 23 (0.99-I.54) & 0.062 & $0.94(0.74-\mathrm{I} .18)$ & 0.581 \\
\hline \multicolumn{6}{|l|}{ Maternal parity } \\
\hline 0 & $486 / 445979$ & I & & I & \\
\hline I & $521 / 361097$ & I.32 (I.I7-I.49) & 0.001 & 1.61 (I.4I-I.84) & 0.001 \\
\hline 2 & $216 / 157653$ & I.2I (I.04-I.43) & 0.016 & $1.62(1.36-1.93)$ & 0.001 \\
\hline $3+$ & $24 I / 95637$ & $1.97(1.69-2.30)$ & 0.001 & $2.76(2.29-3.31)$ & 0.001 \\
\hline \multicolumn{6}{|l|}{ Parental occupation } \\
\hline Professional & $190 / 201049$ & I & & $\mathbf{I}$ & \\
\hline Skilled & $858 / 589761$ & I.5I (I.29-I.77) & 0.001 & 1.35 (I.15-I.58) & 0.001 \\
\hline Unskilled & $416 / 269556$ & $2.10(1.76-2.49)$ & 0.001 & $1.69(1.42-2.03)$ & 0.001 \\
\hline \multicolumn{6}{|l|}{ Period of birth } \\
\hline 1969-74 & $724 / 318406$ & I & & 1 & \\
\hline $1975-80$ & $534 / 365842$ & $\mathrm{I} .10(0.98-\mathrm{I} .25)$ & 0.116 & I.I2 (0.99-I.26) & 0.078 \\
\hline$|98|-86$ & $206 / 376118$ & 1.25 (I.04-I.49) & 0.014 & $\mathrm{I} .27$ (I.06-I.52) & 0.010 \\
\hline
\end{tabular}

Young maternal age was also found to be associated with increased risk of offspring deaths from other causes $(<19$ years: $\mathrm{HR}=1.47,95 \%$ CI 1.33-1.63).

\section{Birth weight}

When analysed as a categorical variable, controlling for gestational age as a continuous variable, individuals of low birth weight $(<2500 \mathrm{~g})$, when compared with the reference group $(3250-3749 \mathrm{~g})$, were at higher risk of suicide $(\mathrm{HR}=1.35,95 \%$ CI 1.05-1.72) and at higher risk of death from other causes $(\mathrm{HR}=1.41,95 \% \mathrm{CI}$ 1.24-1.61).

Similar hazard ratios were observed in both males and females. The hazard ratio for low birth weight was higher in the later period of birth (1981-1986), for which full electronic death records were available $(\mathrm{HR}=2.36,95 \%$ CI $1.30-4.27)$. This is in contrast to the earliest period of birth (1969-1974), for which there was no linkage available to infant or early childhood death records $(\mathrm{HR}=0.93,95 \%$ CI 0.64-1.36), and to the middle period (1975-1980), where there would have been partial linkage to such early deaths $(\mathrm{HR}=1.78$, 95\% CI 1.19-2.65). The population-attributable fraction for the influence of low birth weight on suicide in the entire cohort was 0.013 although, when confined to the 1981-1986 sub-cohort, this rose to 0.03 . When treated as a dichotomous variable, those of low birth weight $(<2.5 \mathrm{~kg})$ were again found to have a higher risk of suicide compared with the rest of the cohort (multivariable $\mathrm{HR}=1.29$, 95\% CI 1.02-1.62). Similarly, when treated as a continuous variable, an inverse relationship between increasing birth weight and risk of suicide was demonstrated (multivariable $\mathrm{HR}=0.9997,95 \%$ CI 0.9996-0.9998, $P=0.001$ ).

However, when gestational age was controlled for as a dichotomous variable, the relationship between low birth weight (as a categorical variable) and suicide was not significant $(\mathrm{HR}=1.26,95 \% \mathrm{CI}$ 0.98-1.61, $P=0.068$ ).

\section{Gestational age}

Treating gestational age as a continuous variable, on multivariable analysis no independent association was found between 
gestational age and either subsequent suicide or death from other causes. Similarly, when gestational age was analysed as a dichotomous variable, no such association was observed.

\section{Parental occupation}

Compared with the reference group of parents in professional occupations, offspring of parents in skilled occupations $(\mathrm{HR}=1.35,95 \%$ CI $1.15-1.58)$ and of parents in unskilled occupations $(\mathrm{HR}=1.69$, $95 \%$ CI $1.42-2.03)$ were more likely to have died by suicide.

\section{DISCUSSION}

\section{Findings}

This study found an association between maternal parity and later suicide of offspring. Mittendorfer-Rutz et al (2004) found a significant association between higher maternal parity and attempted suicide, but this is the first report of an association with completed suicide. A higher incidence of some psychiatric disorders has been demonstrated in the younger children of grandmultiparous women, suggesting that to have six or more elder siblings could be detrimental to mental health (Kemppainen et al, 2000). However, the association reported here was not confined to grandmultiparity. We found a significantly higher risk in those born to mothers who had just one previous completed pregnancy. The hazard ratio for maternal parity increased following multivariable adjustment, possibly reflecting a negative correlation between higher maternal parity and younger maternal age. The effect observed was independent of parental occupation. The associations between both low birth weight and younger motherhood, and subsequent adult suicide, replicate in a Scottish cohort the findings of Mittendorfer-Rutz et al (2004), who used similar methods in a Swedish cohort.

The only proxy of affluence available for this study was parental occupation, and skilled and unskilled occupational groups had increased rates of suicide of offspring compared with professional occupational groups. An association between suicide and economic disadvantage has been documented elsewhere (Gunnell et al, 1995; Taylor et al, 2004), as has a link between suicide attempt and parental economic circumstances (Fergusson et al, 2000).
With attributable fractions in the order of 0.24 for maternal parity and 0.20 for young maternal age, the influence of these variables on offspring suicide is potentially of population significance.

\section{Possible interpretations}

Lucas (1991) described a concept known as programming, whereby a physiological stimulus or insult, operating during a specific developmental window of sensitivity, could result in a long-standing or lifelong effect on the structure or function of an organism. A relationship between foetal nutrition and adult disease has been proposed (Godfrey \& Barker, 2000), and it has been suggested that intra-uterine stressors or later childhood neglect could alter gene expression (Oquendo \& BacaGarcia, 2004).

Suicide is strongly correlated with mental disorders, especially affective disorders (Henriksson et al, 1993). It is likely that any variable associated with increased risk of suicidal behaviour would also be associated with increased vulnerability to mental disorders in general. There is a welldocumented link between mental disorder and increased risk of premature death, not only from suicide but from other causes also. Lawrence et al (2000), Joukamaa et al (2001) and Kisely et al (2005), in population-based studies of psychiatric patients, found the highest cause-specific mortality rate ratio for death from suicide and also significant, but lesser, increases in risk of death from other causes. Our similar findings on the effects of maternal parity and maternal age would therefore be consistent with the interpretation that these variables mediate their effect by influencing susceptibility to mental illness. A maternal-foetal origins hypothesis regarding the aetiology of mood disorders (O'Keane \& Scott, 2005) proposes that maternal stress exerts a negative influence on foetal brain development, possibly mediated by the hypothalamic-pituitaryadrenal axis. Cortisol levels in the foetus correlate with those in the maternal circulation (Gitau et al, 1998). High cortisol levels in utero could have a lifelong influence on the hypothalamic-pituitary-adrenal axis, resulting in hypercortisolaemia in adulthood (Thompson et al, 2001), which has been linked to depressive disorders (Nemeroff et al, 1984). This hypothesis is supported by the findings of O'Connor et al (2002) that maternal anxiety during pregnancy is associated with emotional and behavioural problems in 4-year-old offspring. There is also evidence that high maternal stress in utero increases the risk of low birth weight (Dole et al, 2003), but this evidence is conflicting (Andersson et al, 2004).

Theoretically, it is possible that mothers experience greater stress during pregnancy if they are teenagers, have an older child to care for or are financially disadvantaged. The results reported here are, therefore, consistent with the maternal-foetal origins hypothesis.

Bowlby's theory (1977) that the nature of the affectional bonds made in childhood can influence psychological development could also provide a plausible mechanism for some of the effects demonstrated. There is some evidence that perinatal traumatic stress can influence mother-child interactional behaviour (Muller-Nix et al, 2004). However, there is little evidence that children in larger families, or babies born to teenage mothers (Coley \& Chase-Landsdale, 1998), are more likely to have attachment difficulties, but the amount of published research on this is small.

Maternal mental health may be a factor in the causal pathway between parity and suicide. Multiparous women have been shown to be at higher risk of depression (Brown \& Harris, 1978), and maternal depression has been linked with a higher offspring suicide rate (Agerbo et al, 2002), although this later observation is likely to be at least partially mediated by genetics and hence independent of parity.

Birth-order effects on psychological development, including suicidal behaviour, have attracted some research interest, but overall results have been contradictory (Ernst \& Angst, 1983). Whether our findings relate to birth order or family size, they are not consistent with the view that family ties are, in themselves, protective against suicide (Tomassini et al, 2003). It is likely that any relationship between the composition of the family of origin and adult mental health is mediated by an interplay of environmental and psychological factors. Maternal parity, maternal age and parental occupation may be crude markers for the quality of parenting.

\section{Methodological issues}

This population-based study uses a large sample size, giving high statistical power and yielding information on the relatively 
rare event of suicide, which would be difficult to obtain by other methods. Most previous studies have focused on suicidal ideation or affective disorder, not having sufficient numbers to examine completed suicide.

Some limitations to the study should be pointed out. Data were not available on deaths occurring outside Scotland. This may have biased the study towards finding a higher incidence of suicide among those less likely to have migrated from the country. An estimate of what proportion of the cohort was likely to have been included in our outcome measure is provided by data from a census taken in April 2001. This recorded 988281 persons, aged between 15 and 32 years and resident in Scotland, who gave Scotland as their country of birth (Registrar General for Scotland, 2003). Compared with the 1290215 births registered in Scotland between 1969 and 1986, this suggests that approximately $77 \%$ of the cohort were resident in Scotland in 2001.

Whereas we can quantify this loss to follow-up, it is more difficult to assess whether the variables studied were likely to differ between Scottish-born persons in Scotland and those elsewhere. There is evidence to suggest that some of these variables may be associated with subsequent migration (Martyn et al, 1993; Jones \& Swerdlow, 1996). We therefore cannot exclude the possibility of migration bias.

Data were not available on deaths occurring between mother's discharge from hospital and January 1981. As premature and very low-birth-weight babies are likely to be overrepresented in this group, we may have disproportionately overestimated the number of such individuals at risk of subsequent suicide, biasing our results towards negative findings in the case of birth weight and gestational age. This has been addressed by the separate analysis of data from the sub-cohort born between 1981 and 1986, which does have full linkage to infant and early childhood deaths (in Scotland) and demonstrates a higher hazard ratio for low birth weight in that group.

The data on economic circumstances were based on parental occupation only, as information on postcode area of residence was not included in earlier Scottish Morbidity Record maternity records. This is not equivalent to deprivation indices used in other studies, thus limiting its comparability.

DANIEL V. RIORDAN, MRCPsych, New Craigs Hospital, Inverness; SIVASUBRAMANIAM SELVARAJ, MSc, Centre for Rural Health Research and Policy, Inverness; CAMERON STARK, FFPH, NHS Highland, Inverness; JULIE S. E. GILBERT, BSc, Information Services, NHS National Services Scotland.

Correspondence: Dr D. V. Riordan, New Craigs Hospital, Leachkin Road, Inverness IV3 8NP, UK. Tel.: +4401463 253627; fax: +4401463 704696; Email: vincent.riordan@haht.scot.nhs.uk

(First received 9 September 2005, final revision 6 July 2006, accepted I August 2006)

Inconsistent coverage during the initial years of Scottish Morbidity Record maternity recording may be a potential source of bias. The collection of Scottish Morbidity Record maternity data increased gradually over the period, as more wards and units began to use the scheme. There is no known geographical pattern to the increase, but we were unable to locate information on the reasons for varying rates of uptake, and so cannot exclude biases. There is no apparent reason, however, why this should affect the internal validity of the findings in the cohort, and this is supported by the similar results from the three different periods of birth.

\section{Implications}

Further research is needed to clarify how the risk factors identified mediate their effect. They may be operating by influencing attachment processes, by altering gene expression, through antenatal maternal stress or, indeed, by directly increasing stress and cortisol levels in the infant or young child. Whatever the mechanisms, the factors identified here may not be the most relevant pregnancy-related associates of suicide. Factors such as single parenthood, whether or not the pregnancy was wanted or planned and inter-pregnancy spacing may be more important.

This study used suicide as an outcome measure. It needs to be established to what extent the perinatal factors identified here are associated with psychiatric morbidity in general. How the variables studied influence other specific causes of death also needs to be explored further.

Many initiatives, aimed at tackling youth suicide, focus on the environment in which young adults find themselves in today. These results suggest that paying attention to early childhood, even antenatal environments, may have an impact on suicide rates in years to come.

\section{REFERENCES}

Agerbo, E., Nordentoft, M. \& Mortensen, P. B. (2002) Familial, psychiatric, and socio-economic risk factors for suicide in young people: nested case-control study. BMJ, 325, 74-77.

Andersson, L., Sundstrom-Poromaa, I., Wulff, M., et al (2004) Neonatal outcome following maternal antenatal depression and anxiety: a population based study. American Journal of Epidemiology, 159, 872-88I.

Bowlby, J. (1977) The making and breaking of affectional bonds. I. Aetiology and psychopathology in the light of attachment theory. British Journal of Psychiatry, 130, 201-210.

Brown, G.W. \& Harris, T. O. (1978) Social Origins of Depression: A Study of Psychiatric Disorder in Women. London: Tavistock.

Brown, G.W., van Os, J., Driessens, C., et al (2000) Further evidence of relation between prenatal famine and major affective disorder. American journal of Psychiatry, 157, 190-195.

Coley, R. L. \& Chase-Landsdale, P. L. (1998) Adolescent pregnancy and parenthood: recent evidence and future directions. American Psychologist, 53 152-166.

Dole, N., Savitz, D. A., Hertz-Picciotto, I., et al (2003) Maternal stress and preterm birth. American Journal of Epidemiology, 157, 14-24.

Ernst, C. \& Angst, J. (1983) Birth Order: Its Influence on Personality. New York: Springer-Verlag.

Fergusson, D. M., Woodward, L. J. \& Horwood, L. J. (2000) Risk factors and life processes associated with the onset of suicidal behaviour during adolescence and early adulthood. Psychological Medicine, 30, 23-39.

Gitau, R., Cameron, A., Fisk, N. M., et al (1998) Fetal exposure to maternal cortisol. Lancet, 352, 707-708.

Glover, V. \& O'Connor, T. G. (2002) Effects of antenatal stress and anxiety. Implications for development and psychiatry. British Journal of Psychiatry, 180, 389-391.

Godfrey, J. M. \& Barker, D. J. P. (2000) Fetal nutrition and adult disease. American Journal of Clinical Nutrition, 71, 1344-1352.

Gunnell, D., Peters, T., Kammerling, R., et al (1995) Relation between parasuicide, suicide, psychiatric admissions, and socioeconomic deprivation. BMJ, 3II, 226-230.

Hall, D. J., O'Brien, F., Stark, C., et al (1998) Thirteen-year follow-up of deliberate self-harm, using linked data. British journal of Psychiatry, I72, 239-242.

Hawton, K. \& James, A. (2005) Suicide and deliberate self harm in young people. BMJ, 330, 891-894.

Henriksson, M. M., Aro, H. M., Marttunen, M. J., et al (1993) Mental disorders and comorbidity in suicide. American Journal of Psychiatry, 150, 935-940.

Jones, M. E. \& Swerdlow, A. J. (1996) Bias caused by migration in case-control studies of prenatal risk factors for childhood and adult diseases. American Journal of Epidemiology, 143, 823-831. 
Joukamaa, M., Heliövaara, M., Knekt, P., et al (200I) Mental disorders and cause-specific mortality. British Journal of Psychiatry, 179, 498-502.

Kelly, S. \& Bunting, J. (1998) Trends in suicide in England and Wales, 1982-1996. Population Trends, 92 $29-41$.

Kemppainen, L., Makikyro, T., Jokelainen, J., et a (2000) Is grandmultiparity associated with offspring's hospital-treated mental disorders? A 28-year follow-up of the North Finland 1966 birth cohort. Social Psychiatry and Psychiatric Epidemiology, 35, 104-108.

Kendrick, S. \& Clarke, J. (1993) The Scottish record linkage system. Health Bulletin, 5I, 72-79.

Kisely, S., Smith, M., Lawrence, D., et al (2005) Mortality in individuals who have had psychiatric treatment. Population-based study in Nova Scotia. British Journal of Psychiatry, 187, 552-558.

Lawrence, D., Jablensky, A.V., Holman, C. D. J., et al (2000) Mortality in Western Australian psychiatric patients. Social Psychiatry and Psychiatric Epidemiology, 35, 34I-347.

Li, C. I., Daling, J. R. \& Emanuel, I. (2003) Birthweight and risk of overall and cause-specific childhood mortality. Paediatric and Perinatal Epidemiology, I7, 164-170
Lucas, A. (1991) Programming by early nutrition in man. In The Childhood Environment and Adult Disease (eds G. R. Bock \& J.Whelan), pp. 138-155. Chichester: John Wiley \& Sons.

Martyn, C. N., Barker, D. J. \& Osmond, C. (1993) Selective migration by birthweight. Journal of Epidemiology and Community Health, 47, 76.

Mittendorfer-Rutz, E., Rasmussen, F. \& Wasserman, D. (2004) Restricted fetal growth and adverse materna psychosocial and socioeconomic conditions as risk factors for suicidal behaviour of offspring: a cohort study. Lancet, 364, II35-1140.

Muller-Nix, C., Forcada-Guex, M., Pierrehumbert, B., et al (2004) Prematurity, maternal stress and mother-child interactions. Early Human Development, 79. $145-158$

Nemeroff, C. B., Widerlov, E., Bisette, G., et al (1984) Elevated concentrations of CSF corticotropin-releasing factor-like immuno-reactivity in depressed patients. Science, 226, 1342-1344.

O'Connor, T. G., Heron, J., Golding, J., et al (2002) Maternal antenatal anxiety and children's behavioural/ emotional problems at 4 years. Report from the Avon Longitudinal Study of Parents and Children. British Journal of Psychiatry, 180, 502-508.
O'Keane, V. \& Scott, J. (2005) From obstetric complications' to a maternal-foetal origin hypothesis of mood disorder. British Journal of Psychiatry, 186 367-368.

Oquendo, M. A. \& Baca-Garcia, E. (2004) Nature versus nurture: evidence of intrauterine effects on suicidal behaviour. Lancet, 364, |102-1104.

Registrar General for Scotland (2003) Scotland's Census 200I, Reference Volume. Edinburgh: General Register Office for Scotland.

Taylor, R., Page, A., Morrell, S., et al (2004) Socioeconomic differentials in mental disorders and suicide attempts in Australia. British Journal of Psychiatry, 185, 486-493.

Thompson, C., Syddall, H., Rodin, l., et al (200I) Birth weight and the risk of depressive disorder in late life. British Journal of Psychiatry, 179, 450-455.

Tomassini, C., Juel, K., Holm, N., et al (2003) Risk of suicide in twins: 51 year follow up study. BMJ, 327, 373-374.

World Health Organization (1977) International Statistical Classification of Diseases and Related Health Problems, Ninth Revision (ICD-9). Geneva: WHO.

World Health Organization (1992) Internationa Statistical Classification of Diseases and Related Health Problems, Tenth Revision (ICD-10). Geneva: WHO. 